\title{
Knowledge Construction with Causal Concept Maps in a Teachable Agent Environment
}

\author{
James R. Segedy, John S. Kinnebrew, and Gautam Biswas \\ Vanderbilt University, Nashville, TN 37235, USA \\ \{James.R. Segedy, John.S.Kinnebrew, Gautam. Bi.swas\} @vanderbilt. edu
}

We have developed Betty's Brain [1], a computer-based learning environment that employs the learning-by-teaching paradigm to foster students' acquisition of science knowledge and self-regulated learning strategies. The system provides students with opportunities for self-directed, open-ended learning in science. In this learning environment, students are given a knowledge construction task in which they teach a virtual agent by engaging in an iterative process of reading source material and structuring their knowledge in a causal concept map for a particular science domain (e.g., ecology or thermo-regulation). The agent, then, can use this map to answer questions and take quizzes.

The act of teaching an agent is a self-directed and open-ended activity where one explores, integrates, and structures knowledge first for oneself, and then for others. Our previous work has shown that students are motivated to teach and interact with their teachable agent, and this motivation can further enhance their learning.

The learning process is augmented with social interactions. Both the teachable agent and a knowledgeable mentor agent provide conversational feedback about the student's progress and activity patterns. These restricted, popup-based conversations help students (1) understand the science topic, (2) build the correct concept map, and (3) acquire general-purpose problem-solving and metacognitive strategies.

This interactive event will showcase some of the key features of the Betty's Brain system by allowing participants to teach a causal concept map about global climate change. First, they will gain familiarity with the overall task of knowledge construction and map-building in the system: they will search hypertext resources for causal relationships and use them to construct a map. Second, they will learn about monitoring features for exploring their own knowledge explicitly. They will accomplish this by: asking their teachable agent to explain how to answer questions involving complex chains of reasoning, asking their agent to take a quiz, and taking notes about what they know and don't know. Finally, they will encounter conversational dialog from the agents to prompt them to monitor and regulate their own learning along the way.

\section{Reference}

1. Biswas, G., Leelawong, K., Schwartz, D., Vye, N., Vanderbilt, T.: Learning by teaching: A new agent paradigm for educational software. Applied Artificial Intelligence 19(3), 363-392 (2005) 